\title{
Effect of Increased Intra-abdominal Pressure on Liver Histology and Hemodynamics: An Experimental Study
}

\author{
EFSTATHIOS A. ANTONIOU ${ }^{1}$, EVI KAIRI ${ }^{2}$, GEORGIOS A. MARGONIS ${ }^{3}$, NIKOLAOS ANDREATOS ${ }^{3}$, \\ KAZUNARI SASAKI ${ }^{3}$, CHRISTOS DAMASKOS ${ }^{1}$, NIKOLAOS GARMPIS ${ }^{1}$, MARIO SAMAHA ${ }^{3}$, \\ ERIPHYLI ARGYRA ${ }^{4}$, GEORGE POLYMENEAS ${ }^{5}$, MATTHEW J. WEISS ${ }^{3}$, \\ TIMOTHY M. PAWLIK ${ }^{3}$, DIONYSIOS VOROS ${ }^{5}$ and GREGORY KOURAKLIS ${ }^{1}$ \\ ${ }^{1}$ Second Department of Propaedeutic Surgery, Laiko Hospital, University of Athens, Athens, Greece; \\ ${ }^{2}$ Department of Pathology, ${ }^{4}$ First Department of Anesthesiology, and ${ }^{5}$ Second Department of Surgery, \\ Aretaieion Hospital, University of Athens School of Medicine, Athens, Greece; \\ ${ }^{3}$ Department of Surgery, The Johns Hopkins University School of Medicine, Baltimore, MD, U.S.A.
}

\begin{abstract}
Background: While reduction of portal venous $(P V)$ blood flow has been described in animal models of intra-abdominal hypertension, reports on compensatory changes in hepatic arterial (HA) flow, known as the hepatic arterial buffer response are controversial. Materials and Methods: Pneumoperitoneum with helium was induced in 13 piglets. Hemodynamic measurements and pathological assessment were conducted at baseline and during the three subsequent phases: Phase A: 45 minutes with a stable intraabdominal pressure of $25 \mathrm{mmHg}$; phase B: 45 minutes with a stable intra-abdominal pressure of $40 \mathrm{mmHg}$; and phase $C$ during which the abdomen was re-explored and reperfusion of the liver was allowed to take place. Results: Phase B pressure was significantly greater than phase A pressure in both the $P V$ and the inferior vena cava, demonstrating a positive association between escalating intra-abdominal hypertension and the pressure in these two vessels (all $p<0.001)$. In contrast, HA pressure was comparable between baseline and phase A, while it tended to decrease in phase B. Regarding histology, the most notable abnormality was the presence of inflammatory infiltrates and hepatocyte necrosis. Conclusion: Heliuminsufflation increased $P V$ pressure with a partial
\end{abstract}

This article is freely accessible online.

Correspondence to: Gregory Kouraklis, MD, PhD, Professor in Surgery and Director of 2nd Department of Propaedeutic Surgery, Chairman of Surgery at the School of Medicine, University of Athens, University of Athens, Medical School, 122 Vasilisis Sofias Avenue, Athens, 11526, Greece. Tel: +30 2107456668, Fax: +30 2107456972, e-mail: gkouraklis@hotmail.com

Key Words: Intra-abdominal hypertension, porcine model, liver histology and hemodynamics. compensatory decrease of HA pressure. Nonetheless, findings consistent with hepatic ischemia were observed on pathology.

Intra-abdominal hypertension (IAH) and abdominal compartment syndrome (ACS) are closely related clinical entities that are known to develop in critically-ill patients, following major surgery, trauma, or large volume fluid resuscitation $(1,2)$. Both conditions are part of the same pathophysiological continuum characterized by increased intra-abdominal pressure and subsequent compression of intra-abdominal organs and vascular structures and, as such, they may not be truly distinct; however, the need for methodological consistency and standardized patient management necessitates their precise definition (3). Consequently, in a recent expert consensus statement, IAH was defined as sustained intra-abdominal pressure that exceeds $12 \mathrm{~mm} \mathrm{Hg}$; ACS, on the other hand, was defined as the concurrent presence of significant IAH $(>20 \mathrm{~mm} \mathrm{Hg})$ and end-organ dysfunction (1). Even with these uniform definitions in place, however, the true prevalence of both conditions remains difficult to estimate. For example, according to a recent systematic review, the reported incidence of ACS among patients with traumatic injury ranged from $0 \%$ to $36.4 \%$ in the published literature (4). Similarly, conflicting estimates have been published with respect to the true prevalence of IAH and ACS in the general medical/surgical population as well as in patients with burns $(2,3,5)$. Nonetheless, given the potential for staggering mortality rates that may range from $39-55 \%$, the importance of prompt diagnosis and effective management of both conditions has never been controversial (2-4).

The pathophysiological sequelae responsible for the grim prognosis of IAH and ACS have been studied extensively in both experimental and clinical settings (2, 3, 6-11). 
Interestingly, IAH has been demonstrated to be a truly systemic condition, whose effects range from decreased cardiac output, impaired abdominal perfusion and reduced lung capacity to compromised renal function and even increased intracranial pressure $(7,12-17)$. Consequently, it is not surprising that IAH has also been shown to have detrimental effects on the liver. Specifically, the presence of IAH is known to reduce the blood flow in both the hepatic artery (HA) and the portal vein (PV), thus compromising the liver's physiological capacity to regulate its blood flow (9, 18). Furthermore, along with these hemodynamic alterations, IAH has also been shown to result in varying degrees of hepatocyte necrosis, as well as impaired hepatic energy metabolism (19-21). Interestingly, however, the effects of progressively worsening IAH on liver hemodynamics and the hepatic parenchyma have not been well studied. As this issue may have significant clinical relevance for the management of patients with deteriorating IAH, we aimed to clarify the impact of progressive increases in intra-abdominal pressure on the liver by utilizing a porcine model designed to simulate the effects of IAH.

\section{Materials and Methods}

All experiments were conducted at the 'Kostas Tountas' Experimental Laboratory of Aretaieion University Hospital, which is, in turn, administered by the Second Department of Surgery of the National and Kapodistrian University of Athens (Faculty of Medicine). The study was designed and carried out according to the pertinent national and European legislation on animal care and experimental research and was approved by the Institution's Review Board (approval no.: 4809111999). A certified veterinarian was present during all phases of the study to facilitate appropriate care of the experimental subjects.

Thirteen piglets of both sexes with a mean weight of $26 \mathrm{~kg}$ (range $=25-27 \mathrm{~kg}$ ) were originally enrolled in the study. The animals were denied access to food for 24 hours prior to the experiment, but were allowed to ingest water without restriction. Of the original study population, one animal was used as a pilot in order to standardize the experimental procedure, while four others died as a result of intra-experimental complications before meaningful measurements could be obtained. As such, eight animals were ultimately included in the study population.

Anesthesia and instrumentation. Approximately 30 minutes before the initiation of the experiment, the animals were pre-medicated intramuscularly with $10 \mathrm{mg} / \mathrm{kg}$ ketamine as well as $0.5 \mathrm{mg} / \mathrm{kg}$ diazepam to facilitate their transfer to the operating room. Subsequently, the posterior auricular vein was catheterized (20-G catheter) and basic anesthetic monitoring (electrocardiogram, oxygen saturation, end-expiratory $\mathrm{CO}_{2}$ measurement) was initiated.

Anesthesia was induced with a loading, intravenous (i.v.) dose of $5 \mathrm{mg} / \mathrm{kg}$ sodium pentothal and maintained with i.v. infusion of sodium pentothal at a dose of $1 \mathrm{mg} / \mathrm{kg} / \mathrm{h}$. The animals were intubated with the use of a straight laryngoscope blade and 5-5.5 Fr endotracheal tubes were utilized, as appropriate. Analgesia was achieved with the use of fentanyl $(5 \mu \mathrm{g} / \mathrm{kg} / \mathrm{h})$, while pancuronium was used as a muscle relaxant (loading dose of $0.1 \mathrm{mg} / \mathrm{kg}$ followed by continuous i.v. infusion of $0.3 \mathrm{mg} / \mathrm{kg} / \mathrm{h}$ ). A nasogastric tube was then inserted to both drain the stomach and enable Phi monitoring. An additional $8 \mathrm{Fr}$ transesophageal tube was also inserted to monitor the temperature and intraluminal pressure of the esophagus. Following intubation, the animals were mechanically ventilated with a mixture containing both oxygen/air $\left(\mathrm{FiO}_{2}=60 \%\right)$ and the inhaled anesthetic agent isoflurane in concentrations ranging from $0.5 \%$ to $1.5 \%$. Tidal volume was set at $10-15 \mathrm{ml} / \mathrm{kg}$ and respiratory rate at $15-20 /$ minute. The respiratory rate, in particular, was appropriately modified throughout the experiment so as to maintain an endexpiratory $\mathrm{CO}_{2}$ concentration within the range of $35-40 \mathrm{mmHg}$. Crystalloids were administered at a rate of $10 \mathrm{ml} / \mathrm{kg} / \mathrm{h}$ during the experiment, but the rate of infusion was increased to $20 \mathrm{ml} / \mathrm{kg} / \mathrm{h}$ when the intra-abdominal pressure was raised to $40 \mathrm{mmHg}$, so as to maintain systemic perfusion. Colloids were also administered for the same reason during this stage of the experiment.

In addition, the right internal jugular vein and right internal carotid artery were surgically exposed and catheterized. Specifically, a $5.5 \mathrm{Fr}$ Swan-Ganz catheter $(75 \mathrm{~cm})$ was inserted into the internal jugular vein and used to continuously monitor pulmonary artery (PA) pressure, pulmonary wedge pressure and cardiac output (the latter using the calibration in vivo technique). The right internal carotid artery was catheterized with a $20-\mathrm{G}$ arterial catheter, which was used to continuously monitor blood pressure as well as to obtain blood samples, as dictated by the experimental protocol. Following laparotomy and before the start of the various experimental phases, the common HA was catheterized through the gastroduodenal artery with a $20-$ to $22-\mathrm{G}$ catheter $(35 \mathrm{~cm})$. The inferior mesenteric and femoral veins were also surgically exposed at this time and were used to catheterize the PV and inferior vena cava (IVC), respectively. These catheters were used to monitor the pressure of the aforementioned vessels, during the various experimental phases.

Experimental phases. After the abdominal cavity of the animals was entered (Figure 1A) and the previously discussed instrumentation completed, a baseline arterial blood gas sample was collected. Baseline measurements of heart rate (HR), and arterial, central venous (CV), HA, PV, IVC (measured at the level of the hepatic veins), PA and PA wedge pressure were also performed. At this time, the liver was also surgically biopsied (Figure 1B). Following the completion of these baseline measurements, a Verres needle was employed to induce a pneumoperitoneum and the abdomen of the animals was closed with continuous Nylon 2 sutures. Following that, the experiment was divided into three distinct phases according to the level of induced intra-abdominal pressure: Phase A: 45 minutes with a stable intra-abdominal pressure of $25 \mathrm{mmHg}$; phase B: 45 minutes with a stable intra-abdominal pressure of $40 \mathrm{mmHg}$; phase $\mathrm{C}$ : a final phase during which the abdomen was re-explored and reperfusion of the liver was allowed to take place. The aforementioned hemodynamic measurements were repeated at the end of each experimental phase, as was the drawing of blood samples, so as to provide a means of comparing the effects of different levels of intraabdominal pressure. Finally, two additional surgical biopsies of the liver were performed: a) Immediately after the abdomen was reopened following the phase B (Figure 1C) and b) 15 minutes later, when hepatic reperfusion had macroscopically taken place. The microscopic findings noted in these specimens were then compared with those of the baseline specimen by an experienced pathologist. 

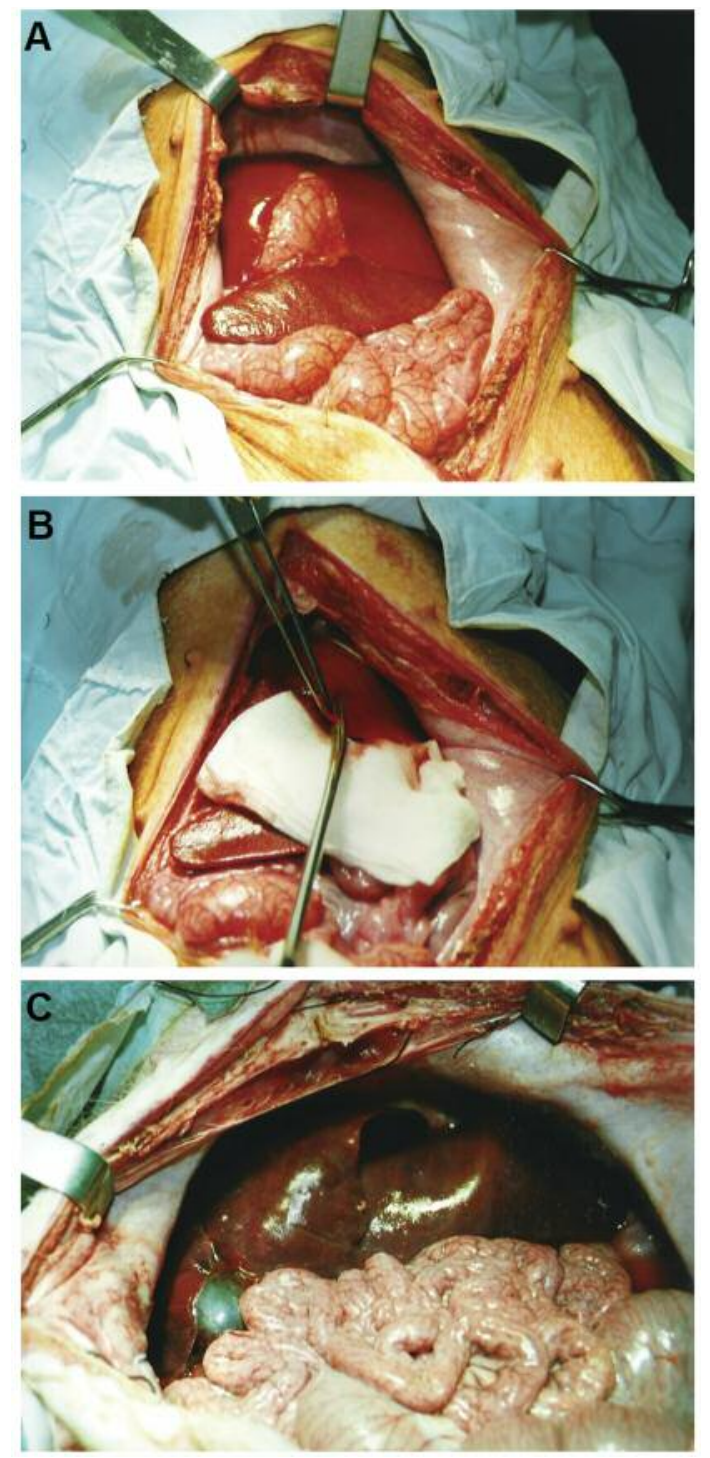

Figure 1. Intraoperative view. A: Opening of abdominal wall. B: Liver biopsy at baseline. $C$ : Liver at the end of phase B.

Statistical analysis. All measured clinical variables were compared over the various time points of the experiment with the use of oneway analysis of variance (ANOVA). The least significant difference (LSD) test was used for post-hoc comparisons. A value of $p<0.05$ was considered statistically significant. All statistical analyses were performed with the aid of STATISTICA for Windows, Rel. 4,5 statistical software (StatSoft Inc., Tulsa, OK. USA).

\section{Results}

The impact of IAH on liver histology. The baseline hepatic specimen, obtained before the initiation of the pneumoperitoneum was remarkable for a limited number of
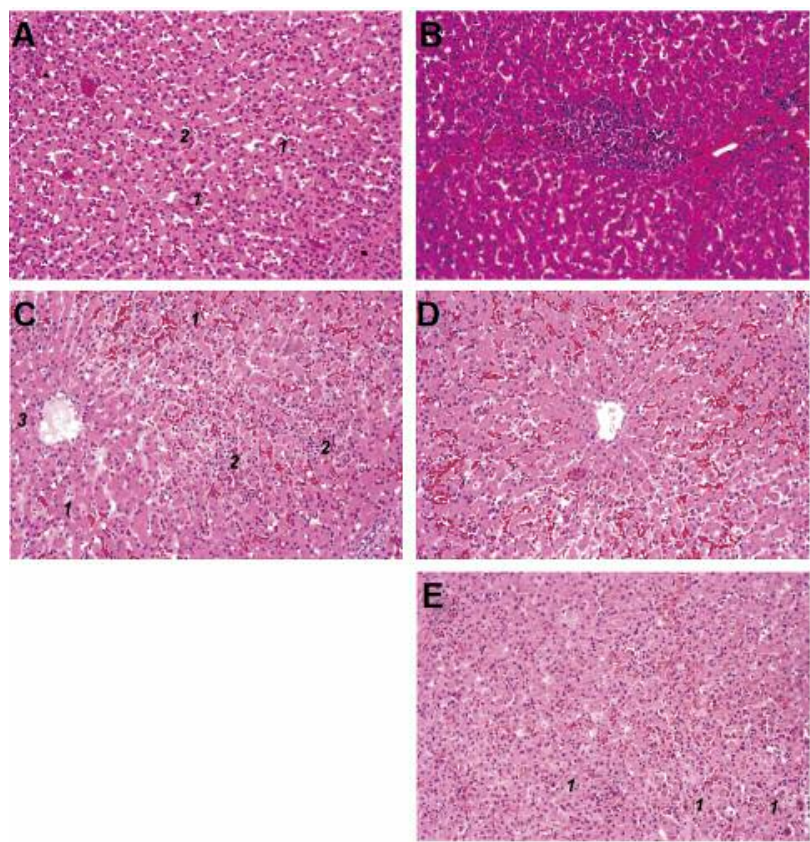

Figure 2. Liver histology at the different phases during surgery. A: At baseline, showing a central vein, slight sinusoidal dilatation (1) and small degree of denudation of sinusoidal endothelial cells (2). B: At baseline, showing inflammatory cells in the periportal space. $C$ : At the end of phase $B$, showing sinusoidal dilatation and hyperemia (1), polymorph aggregates (2) and dilatation of central vein (3). D: At the end of phase $B$, showing dilatation of central vein, sinusoidal dilatation and hyperemia as well as Kupffer cell apoptosis. E: At the end of phase B, showing (1) hepatocyte necrosis. Of note, no hepatic edema was observed.

abnormalities on microscopic examination. Specifically, minimal periportal and interlobular inflammation was noted, as well as mild to moderate distention of the portal venous branches with concomitant inflammation and focal detachment of endothelial cells (Figure 2A and B). Similar findings were noted among the hepatic venous branches and hepatic sinusoids. On the other hand, the hepatocytes themselves appeared microscopically normal with no evidence of necrosis and only rare inflammatory infiltrates. Despite the abnormalities, however, it was the opinion of the examining pathologist that the specimen was essentially normal and the aforementioned findings were probably a result of intra-operative manipulation of the liver.

A substantially different picture emerged following the microscopic examination of the second liver biopsy, obtained immediately after the end of the second phase of the induced pneumoperitoneum $(40 \mathrm{mmHg})$. Specifically, the hepatic capsule was found to contain multiple hemorrhagic and polymorphonuclear inflammatory infiltrates, while moderate to severe distension was noted in the hepatic sinusoids 
in vivo $32: 85-91(2018)$

Table I. Means and standard deviations (in parentheses) of all recorded variables recorded during the four experimental phases.

\begin{tabular}{lccccc}
\hline & Baseline & Phase A $(25 \mathrm{mmHg})$ & Phase B (40 mm Hg) & Phase C $(0 \mathrm{mmHg})$ & $p$-Value \\
\hline Arterial pressure & $87.600(10.600)$ & $96.000(6.000)$ & $87.600(19.500)$ & $72.900(21.800)$ & 0.05 \\
Heart rate (bpm) & $133.000(29.100)$ & $153.600(32.800)$ & $162.900(31.900)$ & $175.000(35.200)$ & 0.088 \\
Central venous pressure (mmHg) & $3.800(3.200)$ & $4.300(2.800)$ & $5.500(2.600)$ & $4.000(3.300)$ & 0.651 \\
Arterial blood pH & $7.140(0.126)$ & $7.080(0.105)$ & $7.060(0.093)$ & $7.050(0.187)$ & 0.576 \\
Pulmonary artery pressure (mmHg) & $18.300(2.700)$ & $22.100(4.800)$ & $23.500(6.600)$ & $20.300(4.600)$ & 0.179 \\
Pulmonary wedge pressure (mmHg) & $8.400(3.900)$ & $6.600(3.700)$ & $9.400(2.800)$ & $9.800(4.400)$ & 0.365 \\
Hepatic artery pressure (mmHg) & $82.900(10.900)$ & $87.600(10.800)$ & $80.000(17.000)$ & $52.400(17.000)$ & $<0.001$ \\
Portal vein pressure (mmHg) & $9.600(3.700)$ & $26.300(5.300)$ & $41.400(4.700)$ & $12.300(8.200)$ & $<0.001$ \\
Inferior vena cava pressure (mmHg) & $6.000(4.900)$ & $22.600(4.500)$ & $41.400(9.300)$ & $6.300(1.800)$ & $<0.001$ \\
\hline
\end{tabular}

(Figure 2C). The latter were also remarkable for significant derangement of their microscopic architecture and hyperemia, as well as extensive endothelial cell apoptosis and detachment (Figure 2D). Interestingly, this was not the case for the portal or hepatic venous branches that appeared essentially similar to those at baseline. Furthermore, the degree of periportal and interlobular inflammation was also unchanged from baseline. The hepatocytes, however, demonstrated significant changes, with the most notable abnormality being the presence of multiple inflammatory infiltrates and hepatocyte necrosis (Figure 2E). A mild to moderate degree of microvesicular steatosis was also observed. Interestingly, the pathological examination of the final liver biopsy, obtained after reperfusion of the liver had macroscopically taken place, revealed similar findings to those of the second biopsy, with no discernible trend towards either improvement or worsening of the previously noted abnormalities.

The effect of progressive IAH on liver hemodynamics. As previously described, arterial blood gas samples were collected and measurements of HR, and arterial, CV, HA, PV, IVC (measured at the level of the hepatic veins), PA and PA wedge pressure were undertaken at baseline and at the end of every experimental phase, resulting in measurements at four distinct time points. These results are summarized in Table I. Interestingly, no statistically significant difference in recorded values between the four time points was detected for $\mathrm{HR}, \mathrm{CV}, \mathrm{PA}, \mathrm{PA}$ wedge pressure and arterial blood $\mathrm{pH}$ (all $p>0.050$ ). The result for arterial pressure was borderline significant $(p=0.050)$ in one-way ANOVA and was shown to be significant in post-hoc comparison analysis, with arterial pressure being significantly reduced after abdominal decompression (phase $\mathrm{C}$ ) in comparison with the end of phase A $(p=0.007)$.

On the other hand, significant differences were detected between the various phases of the experiment in HA, PV and IVC pressure (all $p<0.001$ ) (Table I). Specifically, HA pressure was significantly reduced in phase $\mathrm{C}$ in comparison with baseline and phases A and B (all $p<0.001)$. On the other hand, in the case of both PV and IVC pressure, no statistically significant difference was detected between baseline and phase $C$ (all $p>0.050$ ); however, baseline and phase $\mathrm{C}$ pressures were significantly lower than phase $\mathrm{A}$ and $\mathrm{B}$ (all $p<0.001)$ in both the PV and the IVC pressures. Furthermore, phase B pressure was also significantly greater than those in phase A in both the PV and the IVC, thus demonstrating a positive association between escalating IAH and the pressure in these two vessels (all $p<0.001$ ).

\section{Discussion}

In the current study, we found that elevated intra-abdominal pressure can have a severe impact on liver hemodynamics, hepatic perfusion and parenchymal histology, even within a short time frame. Specifically, an increase in PV pressure was recorded that coincided with increased intra-abdominal pressure. Although we did not directly measure PV flow in the present study, it is reasonable to assume that this hemodynamic parameter must have decreased in conjunction with the recorded increase in PV pressure. Even though this finding might appear unsurprising given the pathophysiology of the syndrome, it should be noted that a number of previous studies failed to demonstrate significant changes in PV hemodynamics during the experimental induction of IAH $(22,23)$. Perhaps more importantly from a physiological standpoint, while PV pressure increased in a linear fashion alongside intra-abdominal pressure, HA pressure did not increase. In fact, HA pressure was even noted to decrease slightly when intra-abdominal pressure increased from 25 to $40 \mathrm{mmHg}$. While this finding seems to be paradoxical, it might be explained by the hepatic arterial buffer response (HABR) (24). HABR is an autoregulatory mechanism that serves to maintain total hepatic blood flow within a range of physiological values $(25,26)$. Essentially, HABR consists of mechanisms that counteract a decrease in the flow of any of the two major vessels responsible for 
hepatic perfusion, namely the HA and PV. It is currently thought that this compensatory response may be explained by the 'adenosine washout hypothesis'; specifically, it is known that adenosine is released relatively constantly in the space of Mall that surrounds both portal venules and hepatic arterioles (27). In turn, adenosine concentrations are regulated by washout into the aforementioned hepatic vessels. As such, if PV blood flow decreases, adenosine washout is also curtailed. As may be expected, adenosine levels subsequently increase, leading to dilation of the HA and restoration of hepatic perfusion. As such, a decrease in the flow of the PV as evidenced by an increased PV pressure, would be expected to induce a compensatory increase in the flow/decrease in the pressure of the HA.

In the current study, HA pressure did in fact decrease slightly between the $25 \mathrm{~mm} \mathrm{Hg}$ and $40 \mathrm{mmHg}$ time-points; however, this decrease was not statistically significant and was unable to cancel out the much greater decline in hepatic perfusion caused by the observed increase in PV pressure. Therefore, it appears that HABR is only partially preserved in the setting of IAH. Our findings are in line with those of Ishizaki et al. who found that HA blood flow remained stable during PV flow reduction when intra-abdominal pressure increased to $16 \mathrm{~mm} \mathrm{Hg} \mathrm{(28).} \mathrm{While} \mathrm{the} \mathrm{authors}$ attributed their finding to an intact HABR that serves to preserve HA flow during PV flow reduction, others questioned this interpretation. Specifically, Richter et al. postulated that a truly intact HABR would cause a significant increase in HA blood flow that, in turn, would restore hepatic perfusion (the sum of HA and PV blood flow) to normal (18). Our findings are important because they may help elucidate a controversy in the IAH literature; specifically many investigators have shown that both HA and PV blood flow decrease simultaneously during pneumoperitoneum, while other studies have found no impact of increased intra-abdominal pressure on HA and PV blood flow $(22,29)$. In contrast with our results, others have even found the opposite result, namely a decrease of HA flow and maintenance of PV flow during elevated intraabdominal pressure $(29,30)$. Interestingly, it appears that the choice of animal model can impact the preservation of HABR in the setting of elevated intra-abdominal pressure (29). For example, a study based on a neonatal lamb model demonstrated intact HABR with doubled HA blood flow following induction of IAH; on the contrary HA blood flow was unchanged in a dog model, while an additional study based on a pig model reported both impaired HABR and reduced HA blood flow $(9,28,31)$. The latter result is intriguing, as it directly contradicts our findings.

A possible explanation of these disparate findings might be that in contrast with most other animal models that employ $\mathrm{CO}_{2}$-induced pneumoperitoneum, we used a heliuminduced pneumoperitoneum in the present study.
Specifically, $\mathrm{CO}_{2}$ induced pneumoperitoneum may result in acidosis, a factor known to influence the regulation of hepatic perfusion (32). For example, Richter and colleagues showed that acidosis distorts the autoregulation of hepatic blood flow by diminishing HABR (18). In addition, Fujita et al. also demonstrated that acidosis significantly influences hepatic perfusion (33). Nonetheless, it should be noted that the use of a helium-induced pneumoperitoneum can theoretically still result in acidosis, if it is associated with splanchnic hypoperfusion. However, we do not believe that this occurred in the present study, as $\mathrm{pO}_{2}$ levels remained in the normal range throughout the entire experiment. As such, it appears that the animals were protected from acidosis; in turn, this might explain why HABR was at least partially maintained.

In line with the changes in PV blood flow, hepatic function, as noted in the histological examination, was also impaired. Interestingly, while the degree of periportal and interlobular inflammation was unchanged from baseline, the hepatocytes demonstrated significant changes, with the most notable abnormality being the presence of multiple inflammatory infiltrates and hepatocyte necrosis. These findings are in line with those of other investigators who used liver enzymes such as aspartate aminotransferase and alanine aminotransferase as surrogates of liver function and found that their serum activities were increased as a result of increased intra-abdominal pressure $(34,35)$. Of note, while IVC pressure was elevated in a similar fashion to $\mathrm{PV}$ pressure, there was no histological evidence of reduced liver outflow. Specifically, there were no signs of acute hepatic venous outflow obstruction such as sinusoidal congestion or cell necrosis in the perivenular areas of the hepatic acini. A possible explanation for this finding, might be that the reduced liver inflow (because of the reduced PV flow) effectively 'cancelled out' the reduced venous outflow that accompanied the elevation in IVC pressure, thus resulting in no significant histological changes.

In conclusion, the present study demonstrated that IAH is accompanied by a linear increase in PV pressure which, in turn, reduces liver inflow. Unlike previous studies, we found that HABR, an intrinsic hepatic blood flow regulatory system, is maintained during IAH, at least to an extent. This finding might be partially attributed to the helium-induced pneumoperitoneum that constitutes a unique feature of our experimental model. Despite the relative preservation of HABR, our histological findings were compatible with ischemia as a result of decreased PV blood flow. Given that in current clinical practice lower-pressure (e.g. 10-12 mmHg) pneumoperitoneum is no longer considered sufficient to maintain the necessary intra-abdominal space for surgical procedures, future studies are needed to elucidate the physiology of HABR with the aim of preserving this important regulatory mechanism during laparoscopic operations. 


\section{Conflicts of Interest}

None to report

\section{Acknowledgements}

The Authors would like to thank Ms. Nefeli Bampatsikou for excellent technical support.

\section{References}

1 Kirkpatrick AW, Roberts DJ, De Waele J, Jaeschke R, Malbrain ML, De Keulenaer B, Duchesne J, Bjorck M, Leppaniemi A, Ejike JC, Sugrue M, Cheatham M, Ivatury R, Ball CG, Reintam Blaser A, Regli A, Balogh ZJ, D'Amours S, Debergh D, Kaplan M, Kimball E, Olvera C and Pediatric Guidelines SubCommittee for the World Society of the Abdominal Compartment $\mathrm{S}$ : Intra-abdominal hypertension and the abdominal compartment syndrome: updated consensus definitions and clinical practice guidelines from the World Society of the Abdominal Compartment Syndrome. Intensive Care Med 39: 1190-1206, 2013.

2 Carr JA: Abdominal compartment syndrome: a decade of progress. J Am Coll Surg 216: 135-146, 2013.

3 Maluso P, Olson J and Sarani B: Abdominal compartment hypertension and abdominal compartment syndrome. Crit Care Clin 32: 213-222, 2016.

4 Strang SG, Van Lieshout EM, Van Waes OJ and Verhofstad MH: Prevalence and mortality of abdominal compartment syndrome in severely injured patients: A systematic review. J Trauma Acute Care Surg 81: 585-592, 2016.

5 Cheatham ML, Malbrain ML, Kirkpatrick A, Sugrue M, Parr M, De Waele J, Balogh Z, Leppaniemi A, Olvera C, Ivatury R, D'Amours S, Wendon J, Hillman K and Wilmer A: Results from the International Conference of Experts on Intraabdominal Hypertension and Abdominal Compartment Syndrome. II. Recommendations. Intensive Care Med 33: 951962, 2007.

6 Barnes GE, Laine GA, Giam PY, Smith EE and Granger HJ: Cardiovascular responses to elevation of intra-abdominal hydrostatic pressure. Am J Physiol 248: R208-213, 1985.

7 Diebel LN, Dulchavsky SA and Wilson RF: Effect of increased intra-abdominal pressure on mesenteric arterial and intestinal mucosal blood flow. J Trauma 33: 45-48; discussion 48-49, 1992.

8 Diebel L, Saxe J and Dulchavsky S: Effect of intra-abdominal pressure on abdominal wall blood flow. Am Surg 58: 573-575; discussion 575-576, 1992.

9 Diebel LN, Wilson RF, Dulchavsky SA and Saxe J: Effect of increased intra-abdominal pressure on hepatic arterial, portal venous, and hepatic microcirculatory blood flow. J Trauma 33: 279-282; discussion 282-273, 1992.

10 Cullen DJ, Coyle JP, Teplick R and Long MC: Cardiovascular, pulmonary, and renal effects of massively increased intraabdominal pressure in critically ill patients. Crit Care Med 17: 118-121, 1989.

11 Harman PK, Kron IL, McLachlan HD, Freedlender AE and Nolan SP: Elevated intra-abdominal pressure and renal function. Ann Surg 196: 594-597, 1982.
12 Avraamidou A, Marinis A, Asonitis S, Perrea D, Polymeneas G, Voros D and Argyra E: The impact of ischemic preconditioning on hemodynamic, biochemical and inflammatory alterations induced by intra-abdominal hypertension: an experimental study in a porcine model. Langenbecks Arch Surg 397: 1333-1341, 2012.

13 Schachtrupp A, Lawong G, Afify M, Graf J, Toens C and Schumpelick V: Fluid resuscitation preserves cardiac output but cannot prevent organ damage in a porcine model during $24 \mathrm{~h}$ of intraabdominal hypertension. Shock 24: 153-158, 2005.

14 Ridings PC, Bloomfield GL, Blocher CR and Sugerman HJ: Cardiopulmonary effects of raised intra-abdominal pressure before and after intravascular volume expansion. J Trauma 39 : 1071-1075, 1995.

15 Sugrue M, Jones F, Deane SA, Bishop G, Bauman A and Hillman K: Intra-abdominal hypertension is an independent cause of postoperative renal impairment. Arch Surg 134: 1082$1085,1999$.

16 Marinis A, Argyra E, Lykoudis P, Brestas P, Theodoraki K, Polymeneas G, Boviatsis E and Voros D: Ischemia as a possible effect of increased intra-abdominal pressure on central nervous system cytokines, lactate and perfusion pressures. Crit Care 14: R31, 2010.

17 Diebel LN, Dulchavsky SA and Brown WJ: Splanchnic ischemia and bacterial translocation in the abdominal compartment syndrome. J Trauma 43: 852-855, 1997.

18 Richter S, Olinger A, Hildebrandt U, Menger MD and Vollmar B: Loss of physiologic hepatic blood flow control ("hepatic arterial buffer response") during CO2-pneumoperitoneum in the rat. Anesth Analg 93: 872-877, 2001.

19 Nakatani T, Sakamoto Y, Kaneko I, Ando H and Kobayashi K: Effects of intra-abdominal hypertension on hepatic energy metabolism in a rabbit model. J Trauma 44: 446-453, 1998.

20 Toens C, Schachtrupp A, Hoer J, Junge K, Klosterhalfen B and Schumpelick V: A porcine model of the abdominal compartment syndrome. Shock 18: 316-321, 2002.

21 Mogilner JG, Bitterman H, Hayari L, Brod V, Coran AG, Shaoul R, Lurie M, Eldar S and Sukhotnik I: Effect of elevated intraabdominal pressure and hyperoxia on portal vein blood flow, hepatocyte proliferation and apoptosis in a rat model. Eur $\mathbf{J}$ Pediatr Surg 18: 380-386, 2008.

22 Grundel K, Bohm B, Bauwens K, Junghans T and Scheiba R: Influence of acute hemorrhage and pneumoperitoneum on hemodynamic and respiratory parameters. Surg Endosc 12: 809812, 1998.

23 Odeberg S, Ljungqvist O and Sollevi A: Pneumoperitoneum for laparoscopic cholecystectomy is not associated with compromised splanchnic circulation. Eur J Surg 164: 843-848, 1998.

24 Lautt WW: Role and control of the hepatic artery. In: Hepatic circulation in health and disease. New York: Raven Press; 1981.

25 Lautt WW, Legare DJ and Ezzat WR: Quantitation of the hepatic arterial buffer response to graded changes in portal blood flow. Gastroenterology 98: 1024-1028, 1990.

26 Jakab F, Rath Z, Schmal F, Nagy P and Faller J: The interaction between hepatic arterial and portal venous blood flows; simultaneous measurement by transit time ultrasonic volume flowmetry. Hepatogastroenterology 42: 18-21, 1995.

27 Lautt WW, Legare DJ and d'Almeida MS: Adenosine as putative regulator of hepatic arterial flow (the buffer response). Am J Physiol 248: H331-338, 1985. 
28 Ishizaki Y, Bandai Y, Shimomura K, Abe H, Ohtomo Y and Idezuki Y: Safe intraabdominal pressure of carbon dioxide pneumoperitoneum during laparoscopic surgery. Surgery 114: 549-554, 1993.

29 Klopfenstein CE, Morel DR, Clergue F and Pastor CM: Effects of abdominal $\mathrm{CO} 2$ insufflation and changes of position on hepatic blood flow in anesthetized pigs. Am J Physiol 275: H900-905, 1998.

30 Junghans T, Bohm B, Grundel K, Schwenk W and Muller JM: Does pneumoperitoneum with different gases, body positions, and intraperitoneal pressures influence renal and hepatic blood flow? Surgery 121: 206-211, 1997.

31 Masey SA, Koehler RC, Ruck JR, Pepple JM, Rogers MC and Traystman RJ: Effect of abdominal distension on central and regional hemodynamics in neonatal lambs. Pediatr Res 19: 1244$1249,1985$.

32 Takagi S: Hepatic and portal vein blood flow during carbon dioxide pneumoperitoneum for laparoscopic hepatectomy. Surg Endosc 12: 427-431, 1998.
33 Fujita Y, Sakai T, Ohsumi A and Takaori M: Effects of hypocapnia and hypercapnia on splanchnic circulation and hepatic function in the beagle. Anesth Analg 69: 152-157, 1989.

34 Saber AA, Laraja RD, Nalbandian HI, Pablos-Mendez A and Hanna K: Changes in liver function tests after laparoscopic cholecystectomy: not so rare, not always ominous. Am Surg 66: 699-702, 2000

35 Sato K, Kawamura T and Wakusawa R: Hepatic blood flow and function in elderly patients undergoing laparoscopic cholecystectomy. Anesth Analg 90: 1198-1202, 2000.

Received August 17, 2017

Revised September 16, 2017

Accepted September 19, 2017 